\title{
The Combined Effect of Myocardial Infarction and Ischemia on Excitation Wave Propagation in Ventricular Tissue
}

\author{
Cuiping Liang ${ }^{1}$, Kuanquan Wang ${ }^{1}$, Qince $\mathrm{Li}^{1}$, Henggui Zhang ${ }^{1,2}$ \\ ${ }^{1}$ School of Computer Science and Technology, Harbin Institute of Technology, Harbin, Heilongjiang, \\ China \\ ${ }^{2}$ School of Physics and Astronomy, The University of Manchester, Manchester, UK
}

\begin{abstract}
Aims: Previous studies have shown that the infarction and ischemia of cardiac tissue are strongly correlated with incidence of atrial and ventricular tachyarrhythmias. However, so far the combined effect of myocardial infarction and ischemia on the genesis of cardiac arrhythmias has not been fully understood. Therefore, this study aimed to investigate how the coexistence of myocardial infarction and ischemia alters excitation wave propagation.

Methods: The electrophysiology remodeling under ischemia condition was mimicked based on experimental data and incorporated into TPO6 model. Using the constructed $2 D$ and $3 D$ models, we simulated the excitation wave conduction in ventricular tissue under five different conditions: normal, myocardial ischemia under three levels, and myocardial infarction conditions.

Results: Simulation results showed that the conduction velocity and rotor tracks are different in the normal, infarcted and ischemic conditions. In addition, reentry waves are observed in myocardial infarction with the ischemic condition in $2 D$ and $3 D$ models.

Conclusion: Simulation results demonstrate that the coaction of myocardial infarction and ischemia areas increases spatial electrical heterogeneity of ventricular tissue, which may enhance the pro-arrhythmogenic effect.
\end{abstract}

\section{Introduction}

Sudden cardiac death (SCD) is a leading health problem worldwide [1]. A large proportion of SCDs result from ventricular arrhythmia, particularly amongst patients with prior heart damage from myocardial ischemia and infarction $[2,3]$. The occlusion of coronary artery is the main reason of myocardial ischemia, which is divided into 'phase 1A ischaemia' (the first $15 \mathrm{~min}$ ) and 'phase 1B ischaemia' (15-45 min). Then, there is subacute phase of myocardial infarction (MI) [2] (>45 min), when myocardial infarction tissues is always accompanied by fibrotic tissues [4]. Therefore, $20 \%$ random fibrosis cells (inexcitable) were inserted into the myocardial infarction tissues in our 2D and $3 \mathrm{D}$ real ventricular models.

Several studies have investigated the influence of myocardial ischemia or infarction on wave propagation in cardiac tissue. For example, Clayton et al. and Kazbanov et al. researched the effect of global cardiac ischemia on human ventricular fibrillation $[5,6]$. The research of Deng et al. and Arevalo et al. investigated how infarct areas induce arrhythmia in the tissues of patients with myocardial infarction [3,7]. According to the study of Anversa et al., myocardial infarction and ischemia may coexist in the same heart tissues [8]. However, so far their combined effect on the genesis of cardiac arrhythmias has not been fully understood. Therefore, this study aimed to investigate how the coexistence of myocardial infarction and ischemia alters excitation wave propagation. The differences of conduction velocity and rotor in ischemia tissues with that in infarct tissues were investigated in our simulation, which are important for understanding of the myocardial ischemia / infarction-induced arrhythmia mechanism. Furthermore, 2D and 3D real ventricular models with ischemia and infarct areas were designed to uncover combined action of myocardial ischemia and infarction.

\section{Method}

\subsection{Single cell models and modeling methods}

In single cell simulation, we used the TP06 model, which was based on the human experimental data [9].

$$
I_{\text {KATP }}=G_{\text {KATP }} \frac{1}{1+\left(\frac{[A T P]_{i}}{K_{0.5}}\right)^{H}}\left(\frac{\left[K^{+}\right]_{o}}{5.4}\right)^{n}\left(V_{m}-E_{k}\right)
$$

According to the study of Clayton et al., we introduced the formulation of the ATP activated $\mathrm{K}^{+}$current $\mathrm{IK}_{\mathrm{ATP}}$, and modifications to the ionic model of myocardial 
infarction area were implemented, namely: peak sodium current and peak L-type calcium current were decreased $90 \%$ of the original values in the ten Tusscher model in low level ischemia condition [6]. At the same time, we varied $[\mathrm{ATP}]_{\mathrm{i}}$ from a normal value of $6.8 \mathrm{mM}$ to a reduced value of $6.0 \mathrm{mM}$. We elevated $\left[\mathrm{K}^{+}\right]_{\mathrm{o}}$ from its default value of $5.4 \mathrm{mM}$ to a value of $7.5 \mathrm{mM}$. In condition of middle and high level ischemia, peak sodium current and peak Ltype calcium current were decreased $80 \%$ of the original values. The values of $[\mathrm{ATP}]_{\mathrm{i}}$ were reduced to $5 \mathrm{mM}$ and $4.6 \mathrm{mM}$ for middle and high level ischemia conditions, respectively. And, the values of $\left[\mathrm{K}^{+}\right]_{\mathrm{o}}$ were elevated to $8 \mathrm{mM}$ and $11 \mathrm{mM}$ for middle and high level ischemia conditions, respectively. In addition, according to experimental recordings of Deng et al. [3], modifications to the ionic model of myocardial infarction area were implemented, namely: peak sodium current, peak L-type calcium current, peak potassium currents $\mathrm{IKr}$, and IKs were decreased to $38 \%, 31 \%, 30 \%$ and $20 \%$ of the original values in the ten Tusscher model, respectively.

In single cell simulation, we calculated electrophysiology parameters of epicardial cells in normal, ischemia under three levels (low, mid and high) and infarct conditions. Action potentials (APs) were elicited by pre-pacing the models for one hundred times to reach a stable steady state. And 100 stimuli were applied at a PCL of $1000 \mathrm{~ms}$. APD90 was computed as AP duration at 90\% repolarization. RP represented resting potential. APA represented action potential amplitude. And the maximum of depolarization rate $(\mathrm{dV} / \mathrm{dt}$ max $)$ was the important indicator of depolarization velocity.

\subsection{Multicellular 2D and 3D models}

The cell models were incorporated into a parabolic partial differential equation (PDE) to construct monodomain models of cardiac electrophysiology to describe the reaction-diffusion system in simulating cardiac dynamics. The main equation is:

$$
\frac{\partial V_{m}}{\partial t}=\nabla \cdot D \nabla V_{m}-\frac{I_{i o n}}{\mathrm{C}_{m}}
$$

Where $\mathrm{C}_{\mathrm{m}}=1 \mu \mathrm{F} / \mathrm{cm}^{2}$ is the capacitance, $\mathrm{D}$ is the effective diffusion constant, and the value of $\mathrm{D}$ is set to $0.154 \mathrm{~mm}^{2} / \mathrm{ms}, I_{i o n}$ is the total transmembrane current [9].

The spatial resolution in $2 \mathrm{D}$ and $3 \mathrm{D}$ models are as follows: 2D ideal tissue models: $\Delta x=\Delta y=0.25 \mathrm{~mm}$; 2D real tissue models: $\Delta x=\Delta y=0.35 \mathrm{~mm}$; 3D ventricular model with three cell types (endocardial cells : middle cells : epicardial cells $=25: 35: 45)$ [10]: $\Delta x=\Delta y=0.33$ $\mathrm{mm}$; We used 2D ventricular data, from Ventricular Infarct Segmentation challenge 2012 (http://www. cardiacatlas.org/challenges/ventricular-infarctsegmentation/).
In our 2D simulation, we used an isotropic domain size of $1000 \times 1000$ grid points for the $2 \mathrm{D}$ tissue model and $672 \times 644$ grid points for the $2 \mathrm{D}$ ventricular model, which translated into physical sizes of $62500 \mathrm{~mm}^{2}$ and $53014 \mathrm{~mm}^{2}$, respectively. 2D ideal tissue model was stimulated with strength of $-150 \mathrm{pA} / \mathrm{pF}$, duration of $3 \mathrm{~ms}$ at left side of the tissue, and stimulus area is on the left of the 2D tissue with $3 * 0.25 * 1000 * 0.25=187.5 \mathrm{~mm}^{2}$. 2D ventricular model Stimulus area is in the endocardium area with $10 * 10 * 0.35 * 0.35=12.25 \mathrm{~mm}^{2}$. In simulations on $3 \mathrm{D}$ model, we used an isotropic domain size of $325 \times 325 \times 425$ grid points for the $3 \mathrm{D}$ ventricular model. In $3 \mathrm{D}$ ventricular model simulation, all $3 \mathrm{D}$ tissue models were stimulated with the strength of $-120 \mathrm{pA} / \mathrm{pF}$ and the stimulus duration of $2 \mathrm{~ms}$.

\section{Results}

\subsection{Single cell simulation of normal, ischemia and infarct cell}

As a result in single cell simulation, the myocardial infarction zone action potential was characterized by a longer duration, decreased peak amplitude, increased APD90, and decreased maximum of depolarization rate compared to that in the non-ischemic myocardium (as shown in Figure 1), similar to what has been previously reported [3]. For ischemia conditions, from low to high level ischemia, peak amplitude, APD90 and maximum of depolarization rate decreased. All of values were smaller than the value of normal condition. Resting potential elevated from low to high level ischemia.

\subsection{D Ideal Tissue Simulation}

As can be seen in the figure $2 b$, there was different conduction velocity among normal, myocardial ischemia under three levels and myocardial infarction tissues. By computing, conduction velocity of ideal normal tissues with epicardial cells was $69 \mathrm{~cm} / \mathrm{s}$ and myocardial infarction tissue's conduction velocity was $48.5 \mathrm{~cm} / \mathrm{s}$, in agreement with clinical data[11].

For experiments on 2D myocardial ischemia tissues, conduction velocity declined from $60.5 \mathrm{~cm} / \mathrm{s}$ (low level ischemia) to $56 \mathrm{~cm} / \mathrm{s}$ (middle level ischemia). Then, conduction velocity kept consistent from middle level to high level ischemia (as shown in Figure 2a). In spiral wave experiments, the first stimulus is located on the left area of the 2D tissue at $0 \mathrm{~ms}$. And, the second stimulus area is on the left bottom quarter area at 490ms. Results showed that spiral waves in normal tissues were not steady. In infarcted tissues, spiral waves disappeared due to longer APD. Spiral waves were more stable in ischemia conditions compared with normal condition. 


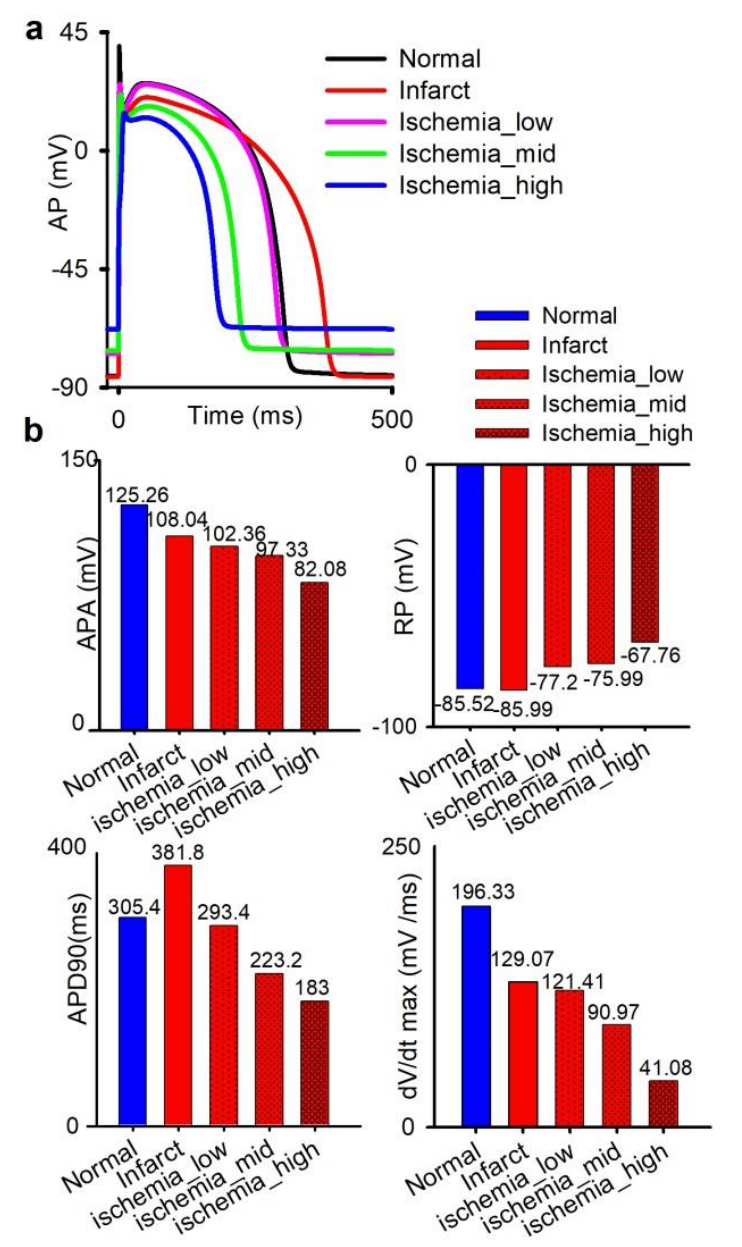

Figure 1. Action potential characteristics of epimyocardial myocytes under normal, ischemia of three levels and infarcted conditions. (a) Action potential of epimyocardial myocytes under normal, ischemia of three levels and infarcted conditions. (b) The value of APA, RP, APD90 and dV/dt max of epimyocardial myocytes under normal, ischemia of three levels and infarcted conditions.

\subsection{Ventricular Tissue Simulation}

In order to research how the coexistence of myocardial infarction and ischemia alters excitation wave propagation, we combine ischemia and infarcted areas into the same ventricular model in 2D and 3D simulation. In consideration of small difference among three levels of ischemia, we just chose middle level of ischemia in our models.

2D ventricular tissues with infarcted areas are from Ventricular Infarct Segmentation challenge 2012. In 3D simulation, firstly 2D scar tissues were inserted, reconstructed and smoothed to form 3D real scar tissues. Then, real 3D scar tissues were put into a 3D human ventricular model as shown in Figure 3(a).

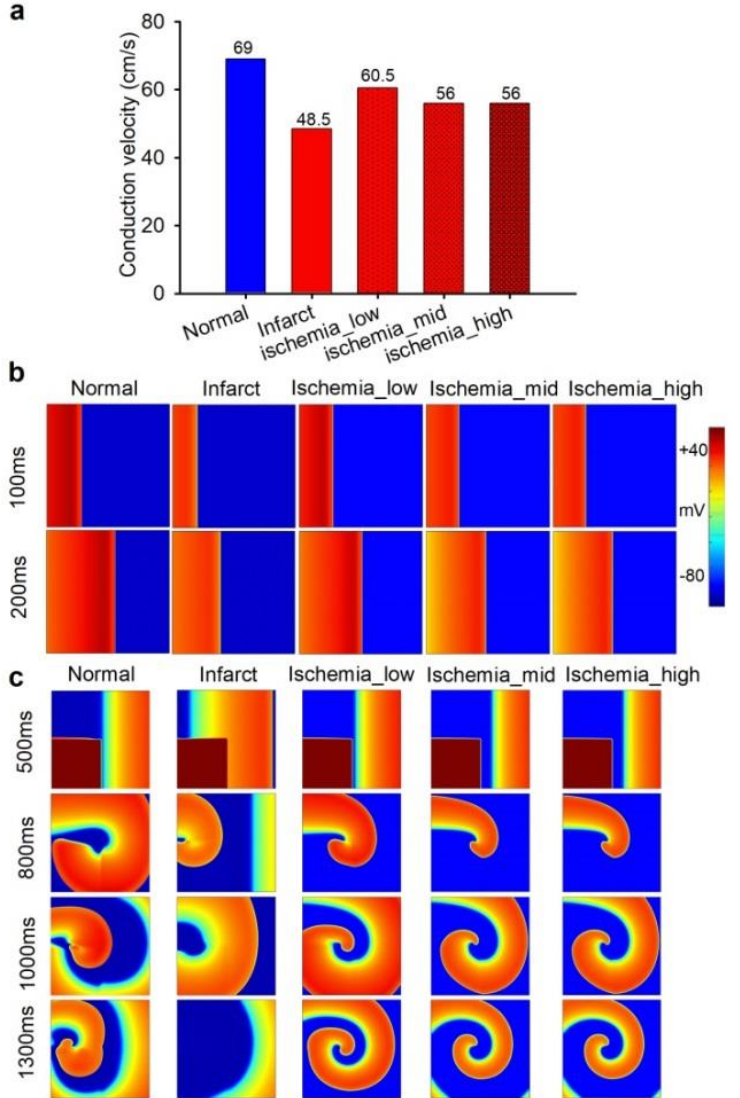

Figure 2. Wave propagation under normal, ischemia of three levels and infarcted conditions in 2D ideal tissues, which is $1000 \times 1000$ grid points with spatial resolution of $0.25 \mathrm{~mm}$. (a) The values of conduction velocity under normal, ischemia of three levels and infarcted conditions. (b) Spiral wave propagation at $100 \mathrm{~ms}$ and $200 \mathrm{~ms}$ under normal, ischemia of three levels and infarcted conditions. (c) Spiral wave propagation at different time under normal, ischemia of three levels and infarcted conditions.

a

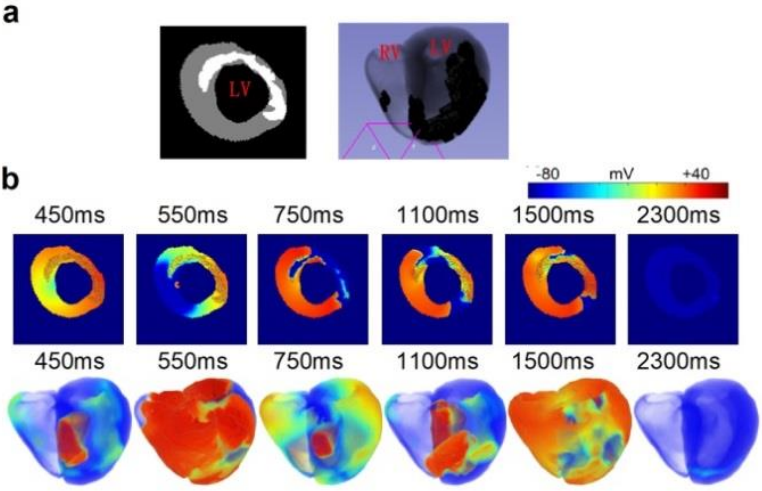

Figure 3. Structural representation and wave propagation in real ventricular models. (a) Structural representation of 2D and 3D real ventricular models. (b) Wave propagation in 2D real ventricular model with ischemia and infarcted areas, which was tested with two stimuli with coupling interval of $540 \mathrm{~ms}$. (c) Wave propagation in $3 \mathrm{D}$ real ventricular model with ischemia 
and infarcted areas, which was tested with three stimuli with coupling intervals of $387 \mathrm{~ms}$ and $720 \mathrm{~ms}$ in turn. (LV: left ventricle; $\mathrm{RV}$ : right ventricle.)

In both simulations of $2 \mathrm{D}$ and $3 \mathrm{D}$ ventricular tissues, reentry waves or scroll waves appeared as shown in Figure 3(b). There were 3 stimuli in 3D simulation, (two stimuli in $2 \mathrm{D}$ simulation), considering that wave length may become shorter with the increasement of stimulus time, which would be beneficial for reentry waves to appear.

\section{Discussion and conclusion}

The aim of this study was to use an electrophysiological model to gain insight into the mechanism underlying the organization of ventricular fibrillation observed in the real ventricular models with cardiac ischemia and infarction.

At the tissue level, in ideal tissue's experiments as shown in Figure 2, conduction velocity of myocardial infarction tissue was lowest $(48.5 \mathrm{~cm} / \mathrm{s})$, comparing with other four conditions: normal and ischemia under three levels. Then, conduction velocity under middle and high level of ischemia conditions both were $56 \mathrm{~cm} / \mathrm{s}$. This is because conduction velocity is more associated with the value of INa. In myocardial infarction condition, the value of INa was smallest (38\% of the original values). Therefore, conduction velocity was smallest. In middle and high levels of ischemia conditions, the values of INa both were $80 \%$ of the original value. Accordingly, the conduction velocity was same in middle and high levels of ischemia conditions. In low level of ischemia condition, the value of $\mathrm{INa}$ is $90 \%$ of the original value. The conduction velocity was $60.5 \mathrm{~cm} / \mathrm{s}$ in this case.

As shown in Figure 2c, spiral waves in normal tissues were little irregular compared with other conditions. In addition, in myocardial infarction condition, spiral waves finally disappeared. This is because longer APD of infarcted area only lead to small part of areas exciting when a second stimulus was applied at same time with other conditions. However, spiral waves were more stable in ischemia conditions, which indicating that ischemia condition was in favor of the steady of spiral waves.

Finally, reentry waves or scroll waves both appeared in simulations of $2 \mathrm{D}$ and $3 \mathrm{D}$ ventricular model with ischemia and infarcted tissues. This is because the coaction of myocardial infarction and ischemia areas increases spatial electrical heterogeneity of ventricular tissue, which may enhance the pro-arrhythmogenic effect.

\section{Acknowledgements}

This work was supported by the National Key R\&D Program of China under Grant 2017YFC0113000.

\section{References}

[1] N. Townsend, L. Wilson, P. Bhatnagar, K. Wickramasinghe, M. Rayner, and M. Nichols, "Cardiovascular disease in Europe: epidemiological update 2016," Eur Heart J, vol. 37, no. 42, pp. 3232-3245, Nov 7, 2016.

[2] J. M. Ferrero, B. Trenor, and L. Romero, "Multiscale computational analysis of the bioelectric consequences of myocardial ischaemia and infarction," Europace, vol. 16, no. 3, pp. 405-15, Mar, 2014.

[3] D. Deng, H. J. Arevalo, A. Prakosa, D. J. Callans, and N. A. Trayanova, "A feasibility study of arrhythmia risk prediction in patients with myocardial infarction and preserved ejection fraction," Europace, vol. 18, no. suppl 4, pp. iv60-iv66, Dec, 2016.

[4] K. H. Ten Tusscher, and A. V. Panfilov, "Influence of diffuse fibrosis on wave propagation in human ventricular tissue," Europace, vol. 9 Suppl 6, pp. vi38-45, Nov, 2007.

[5] I. V. Kazbanov, R. H. Clayton, M. P. Nash, C. P. Bradley, D. J. Paterson, M. P. Hayward, P. Taggart, and A. V. Panfilov, "Effect of global cardiac ischemia on human ventricular fibrillation: insights from a multi-scale mechanistic model of the human heart," PLoS Comput Biol, vol. 10, no. 11, pp. e1003891, Nov, 2014.

[6] R. H. Clayton, M. P. Nash, C. P. Bradley, A. V. Panfilov, D. J. Paterson, and P. Taggart, "Experiment-model interaction for analysis of epicardial activation during human ventricular fibrillation with global myocardial ischaemia," Prog Biophys Mol Biol, vol. 107, no. 1, pp. 101-111, 2011.

[7] H. J. Arevalo, F. Vadakkumpadan, E. Guallar, A. Jebb, P. Malamas, K. C. Wu, and N. A. Trayanova, "Arrhythmia risk stratification of patients after myocardial infarction using personalized heart models," Nat Commun, vol. 7, pp. 11437, May 10, 2016.

[8] P. Anversa, P. Li, X. Zhang, G. Olivetti, and J. M. Capasso, "Ischaemic myocardial injury and ventricular remodelling," Cardiovasc Res, vol. 27, no. 2, pp. 145-57, Feb, 1993.

[9] K. H. ten Tusscher, Panfilov,A.V., "Alternans and spiral breakup in a human ventricular tissue model," Am J Physiol Heart Circ Physiol, vol. 291, no. 3, pp. H1088-100, Sep, 2006.

[10] H. Zhang, S. Kharche, A. V. Holden, and J. C. Hancox, "Repolarisation and vulnerability to re-entry in the human heart with short QT syndrome arising from KCNQ1 mutation--a simulation study," Prog Biophys Mol Biol, vol. 96, no. 1-3, pp. 112-31, Jan-Apr, 2008.

[11] P. Taggart, P. M. Sutton, T. Opthof, R. Coronel, R. Trimlett, W. Pugsley, and P. Kallis, "Inhomogeneous transmural conduction during early ischaemia in patients with coronary artery disease," J Mol Cell Cardiol, vol. 32, no. 4, pp. 62130, Apr, 2000.

Address for correspondence.

Kuanquan Wang.

Mailbox 332, Harbin Institute of Technology

Harbin 150001, China.

wangkq@hit.edu.cn. 